\title{
Health-related quality of life and estimation of minimally important difference in the Functional Assessment of Cancer Therapy-Endocrine Symptom (FACT-ES) score in postmenopausal ER-positive, HER2-negative metastatic breast cancer with low sensitivity to endocrine therapy
}

Yuichiro Kikawa ( $\square$ bj.u1ro@gmail.com )

Kansai Medical University Hospital https://orcid.org/0000-0002-3852-5991

Yasuhiro Hagiwara

The University of Tokyo: Tokyo Daigaku

Tomomi Fujisawa

Gunma Prefectural Cancer Center: Gunma Kenritsu Gan Center

Kazuhiro Araki

Gunma Prefectural Cancer Center: Gunma Kenritsu Gan Center

Takayuki Iwamoto

Okayama University Hospital: Okayama Daigaku Byoin

\section{Takafumi Sangai}

Chiba University Hospital: Chiba Daigaku Igakubu Fuzoku Byoin

\section{Tadahiko Shien}

Okayama University Hospital: Okayama Daigaku Byoin

Shintaro Takao

Hyogo Cancer Center: Hyogo Kenritsu Gan Center

Reiki Nishimura

Kumamoto Shinto General Hospital

Masato Takahashi

NHO Hokkaido Cancer Center

Tatsuya Toyama

Nagoya City University Hospital

Tomohiko Aihara

Aihara Hospital

Hirofumi Mukai

National Cancer Center-Hospital East: Kokuritsu Gan Center Higashi Byoin

Naruto Taira 


\section{Research Article}

Keywords: Health-related quality of life, Breast cancer, Metastasis, Patient-reported outcome measures, Differential threshold

Posted Date: May 27th, 2021

DOI: https://doi.org/10.21203/rs.3.rs-406514/v1

License: (a) (i) This work is licensed under a Creative Commons Attribution 4.0 International License. Read Full License 


\section{Abstract}

Purpose The HORSE-BC study previously demonstrated that 2nd-line endocrine therapy (ET) for metastatic breast cancer (MBC) patients with acquired endocrine resistance still provided a clinically meaningful benefit. Herein, we investigated health-related quality of life (HR-QOL) in HORSE-BC. Methods $M B C$ patients with acquired endocrine resistance who were scheduled for 2nd-line ET were recruited. HRQOL was assessed at baseline and 1 and 3 months after initiation of 2nd-line ET. To investigate the minimally important difference (MID) in the Functional Assessment of Cancer Therapy-Endocrine Symptoms (FACT-ES), we evaluated the means and standard deviations (SDs) for the distribution-based method, and differences in the change in HR-QOL for the anchor-based method. We also investigated the association between FACT-ES total scores and clinical benefit. Results Overall, 56 patients were enrolled. Of these, 47 were analyzed. When defined as 1/3 SD estimates based on the distribution method, the calculated MID was 5.9. The MIDs of the FACT-ES total scores based on the anchor method were 7.7 for decline and 4.1 for improvement. The proportions of MID decline were $6.1 \%$ and $14.7 \%$ lower in patients who experienced clinical benefits than in those who did not at 1 month and 3 months, respectively. The respective ratios of MID improvement in patients who experienced clinical benefits were $18.3 \%$ and $3.2 \%$ higher, respectively; mean change in FACT-ES total score from baseline was improved in patients who experienced clinical benefits. Conclusion Maintaining HR-QOL as determined by FACT-ES may be associated with clinical benefits in patients with acquired endocrine-resistant MBC treated with $E T$.

\section{Introduction}

Endocrine therapy (ET) (plus targeted therapy) is recommended as an initial therapy for patients with hormone receptor (HR)-positive and human epidermal growth factor receptor 2 (HER2)-negative metastatic breast cancer (MBC) without visceral crisis according to the Hortobagyi algorithm and some guidelines [1-3]. Furthermore, subsequent ET should be continued even after patients develop resistance to primary ET because it will not only offer some disease control but will also cause fewer severe adverse events than with chemotherapy. However, the sensitivity to subsequent ET varies among patients, and sometimes, choosing between sequential ET and chemotherapy is difficult, especially for patients assumed to have a low sensitivity to ET. Therefore, we conducted a multicenter prospective observational cohort study (HORSE-BC study) including patients with HR-positive and HER2-negative MBC who were considered as having a low sensitivity to initial ET. Therefore, we concluded that second-line ET might be a viable treatment option for postmenopausal patients with MBC with a low sensitivity to initial ET [4].

In addition to a better clinical response and delayed progression, maintaining the health-related quality of life (HR-QOL) is critical for patients with incurable MBC. HR-QOL is defined as the quantitatively evaluated physical and mental health influenced by a disease or treatment $[5,6]$. It is a multi-dimensional concept comprising fundamental domains, such as functional, physical, psychological, and social domains. To evaluate the HR-QOL, it is important to use patient-reported outcomes (PRO) as a patient's subjective assessment. 
There are studies on the HR-QOL between treatments, which focused on the score differences and variations obtained by questionnaires. However, whether statistically significant differences are clinically important may be unclear. Therefore, it is crucial to interpret the clinically meaningful differences, which are inherent in the score values. As an indicator for interpreting these differences, the minimally important difference (MID) of the HR-QOL score is an essential concept in cancer clinical research $[7,8]$. Although several articles estimating the MIDs of HR-QOL scales used in breast cancer have been published [9-11], there are no studies on the Functional Assessment of Cancer Therapy-Endocrine Symptoms (FACT-ES) [12] in ET for breast cancer. Furthermore, the effect of ethnicity, cultural background, and treatment interventions on MID values in the already estimated HR-QOL score such as FACT-Breast (B) is unclear [13].

The main objectives of the analysis of the HR-QOL in the HORSE-BC study were as follows: First, to clarify the clinically significant scores of the HR-QOL as MIDs associated with ET for MBC. Second, to determine the effect of secondary ET on the HR-QOL in postmenopausal patients with MBC that was less sensitive to primary ET, using the proportion of patients without a decline in the MID.

\section{Patients And Methods}

\section{Study design and participants}

The HORSE-BC study was a multicenter prospective observational study which aimed to evaluate the efficacy and safety of secondary ET among postmenopausal patients with ER-positive and HER2negative MBC who had a low sensitivity to initial ET. Patients who received previous ET as continuous postoperative adjuvant therapy with recurrence within five years after ET initiation or patients who progressed within nine months after initial ET initiation for MBC were included in the study. The other main inclusion criteria were: An Eastern Cooperative Oncology Group performance status (PS) scores of 0-1 and either no previous chemotherapy for MBC or no pre- or post-operative chemotherapy within the past six months. We expected that secondary ET for MBC with a low sensitivity to ET will yield a clinical benefit rate (CBR) of at least $30 \%$ using newer endocrine agents; the expected CBR was $50 \%$. The study results have been described previously [4]. In summary: 1) overall, 56 patients were enrolled, of which 49 were analyzed; 2) the median age was 66 years; 3$) 41$ patients received fulvestrant (82\%), 5 patients received selective estrogen receptor modulators (10\%), 3 patients received a mammalian target of rapamycin inhibitor plus steroidal aromatase inhibitor (AI) (6\%), and 1 patient received Al alone; 4 ) the overall CBR was $44.9 \%$ (90\% confidence interval $(\mathrm{Cl}) 34.6$ to $57.6, \mathrm{P}=0.009)$; and 5 ) the median progression-free survival (PFS) was 7.1 (95\% Cl 5.6 to 10.6) months.

\section{HR-QOL assessment}

HR-QOL was assessed at baseline, 1 month, and 3 months after initiation of second-line ET using the Japanese version of the FACT-General (G), FACT-B, and FACT-ES. The FACT-G measures the general HRQOL associated with cancer using 27 items [7 for physical well-being (PWB), 7 for social and family wellbeing (SFWB), 6 for emotional well-being (EWB), and 7 for functional well-being (FWB)], and the FACT-B 
has 10 additional items on the breast cancer subscale (BCS) that are more specific to women with breast cancer $[13,14]$. The FACT-ES was designed for use with the FACT-B. It is comprised of both 19 items on the endocrine symptom subscale (ESS) and 27 items for the FACT-G, with a possible maximum score of 184 [12]. Each item or question on the FACT-G, B, and ES has response choices ranging from 0 ("not at all") to 4 ("very much"), and then can be converted to total scores and trial outcome index (TOI), which is often used as the main outcome. The FACT-G TOI is the sum of PWB and FWB scores, with a maximum score of 56. The FACT-B TOI is the sum of PWB, FWB, and BCS scores with a maximum score of 96 . The FACT-ES TOI is the sum of PWB, FWB, and ES scores with a maximum score of 132.

\section{Subjective Significant Questionnaire}

To identify MIDs, we provided six questions corresponding to PWB, SFWB, EWB, FWB, ES, and general health as the subjective significant questionnaire (SSQ) [9] at 1 month and 3 months after the start of second-line ET along with the other HR-QOL questionnaires. SSQs are Likert scales with seven grades written as follows: Since you decided to participate in this study, (A) your physical condition is..., (B) your social or relationship with your family people are..., (C) your degree of anxiety is ..., (D) your social activities are..., (E) your menopausal symptoms are..., and (F) your general health status is... The choices after each question are as follows: (1) very much better, (2) moderately better, (3) a little better, (4) about the same, (5) a little worse, (6) moderately worse, and (7) very much worse.

\section{Data analysis}

We analyzed data from patients with baseline HR-QOL assessments. The completion rates for each HRQOL score at each time point were calculated as the number of patients who had each HR-QOL score divided by the number of patients included in the HR-QOL analysis. We summarized the obtained HR-QOL scores at each time point with mean and standard deviation $(\sigma)$. We did not impute missing HR-QOL scores and analyzed only the available scores.

We estimated MIDs of the FACT-G total score and TOI, FACT-B total score and TOI, FACT-ES total score and TOI, and endocrine symptom subscale. To estimate MIDs based on the distribution method [10], we calculated $1 / 2 \sigma, 1 / 3 \sigma$, and the standard error of measurement (SEM) of each score. The SEM was defined as $\sigma(1-\text { rel })^{1 / 2}$, where rel was the reliability of each scale. We used Cronbach's $a$ as a reliability measure in this study. We summarized MID estimates at baseline, 1 month, and 3 months using a weighted mean with the number of scores at each time point as a weight.

The association between the responses to the SSQs and the HR-QOL score changes [9] were used to estimate MIDs based on the anchor method [10]. We used SSQ (E) for the ESS, whereas SSQ (F) was used for the remaining scores. We compared the mean change in the HR-QOL scores of patients with declined (i.e., much worse, moderately worse, or a little worse) and improved (i.e., very much better, moderately better, or a little better) SSQ responses to those with stable (i.e., about the same) SSQ responses using the generalized estimating equation method to account for the correlation between HRQOL scores at 1 and 3 months. We also fitted a dose response model assuming a linear spline in the association between the SSQ responses and changes in HR-QOL scores using the generalized estimation 
equation method. Using the linear spline model with $1 \mathrm{knot}$ at a stable response to the SSQ, we estimated a mean change in the HR-QOL scores corresponding to a moderate and little change in SSQ.

We also investigated the association between the FACT-ES total score and clinical benefit (defined as complete response, partial response, or stable disease for 24 weeks; the primary endpoint in the HORSEBC study). We described cumulative distribution functions at each time point according to the clinical benefit status and compared the proportions of patients who experienced changes in MIDs between the clinical benefit status using the Fisher's exact test. We also compared mean profiles of changes in the FACT-ES total scores according to the clinical benefit status using the generalized estimating equation method.

\section{Results}

\section{Patients}

Of the 56 patients enrolled in the HORSE-BC trial, 7 were excluded based on the inclusion criteria and 2 were excluded due to missing baseline HR-QOL assessments. Finally, 47 patients were included in the HRQOL analysis. The patient characteristics of the QOL/PRO analysis population are shown in Table 1.

\section{Distribution-based MID estimates}

HR-QOL scores and MID estimates based on the distribution-based methods are shown in Table 2. The completion rates were higher than $90 \%$ in all the scores at all time points. For all scores, the smallest distribution-based MID estimates summarized by the weighted mean were $1 / 3 \sigma$, followed in order by SEM and $1 / 2 \sigma$. The distribution-based MID estimates for FACT-B total scores were 8.8 by $1 / 2 \sigma, 5.9$ by $1 / 3 \sigma$, and 6.4 , respectively, by SEM. The FACT-ES total score was 9.2 by $1 / 2 \sigma, 6.2$ by $1 / 3 \sigma$, and 6.7 by SEM, whereas those for FACT-ES TOI were 7.0, 4.6, and 5.3, respectively.

\section{Anchor-based MID estimates}

The liner spline MID estimates by the anchor-based method are shown in Table 3. MID estimates of the FACT-B total scores were 15.9 for moderately worse, 7.9 for a little worse, 2.5 for a little better, and 4.9 for moderately better responses. Those of the FACT-ES total score were 16.7 for moderately worse responses, 8.3 for a little worse responses, 2.0 for a little better responses, and 3.9 for moderately better responses, whereas those for the FACT-ES TOI were 15.7 for moderately worse responses, 7.8 for a little worse responses, 1.5 for a little better responses, and 3.0 for moderately better responses. Furthermore, we categorized the patients into three groups: decline (moderately and a little worse), stable (about the same), and improved (a little and moderately better). These three-category MID estimates based on the anchor-based method are demonstrated in Table 4. For FACT-B, FACT-ES, and FACT-ES TOI, the total scores were 7.3, 7.7, and 7.2 for decline; and 4.4, 4.1, and 3.4 for improvement, respectively. For all scores, MID estimates for decline were larger than the MID estimates for improvement by both the 3-category and 
liner-spline methods. The 3-category MID estimates for decline and improvement were close to slightly worse and moderately better MID estimates by the linear spline method, respectively.

\section{Relationship between clinical benefit and HR-QOL scores or estimated MIDS of FACT-ES}

The cumulative distribution functions for the FACT-ES total score according to the clinical benefit status at 1 and 3 months are shown in Fig. 1a and $\mathbf{b}$, respectively. Using the medians of the estimated distribution-based and anchor-based MIDs ( 8 for decline and 5 for improvement), the proportion of MID decline in the FACT-ES total score at 1 month was $20 \%$ for patients who experienced clinical benefit and $26.1 \%$ for the patients who did not (difference $-6.1 ; 95 \% \mathrm{Cl}-31.2$ to $19 ; \mathrm{P}=0.917$ ). Those at 3 months were $9.1 \%$ and $23.8 \%$ (difference $-14.7 ; 95 \% \mathrm{Cl}-36.5$ to $7.1 ; \mathrm{P}=0.373$ ). The proportions of MID improvement in the FACT-ES total score at 1 month were $40 \%$ for patients who experienced clinical benefit and $21.7 \%$ for patients who did not (difference $18.3 \% ; 95 \% \mathrm{Cl}-9.0$ to $45.6 ; \mathrm{P}=0.333$ ). Those at 3 months were $31.8 \%$ and $28.6 \%$ (difference $3.2 \% ; 95 \% \mathrm{Cl}-24.2$ to $30.7 ; \mathrm{P}=1.0$ ).

The mean changes in the FACT-ES total score from baseline according to the clinical benefit status are shown in Fig. 2. The mean changes in the patients who experienced clinical benefit were $1.3(95 \% \mathrm{Cl}-4.1$ to 6.8$)$ at 1 month and $2.2(95 \% \mathrm{Cl}-1.2$ to 5.6$)$ at 3 months, whereas they were $-4.2(95 \% \mathrm{Cl}-7.9$ to -0.5$)$ at 1 month and $-1.9(95 \% \mathrm{Cl}-6.6$ to 2.8$)$ at 3 months in those who did not. The differences between clinical benefit statuses were $5.5(95 \% \mathrm{Cl} 1.0$ to 12.1$)$ at 1 month and $4.1(95 \% \mathrm{Cl}-1.7$ to 10.0$)$ at 3 months $(P=0.066$ for pooled group difference).

\section{Discussion}

This study investigated the HR-QOL of patients with MBC having a low sensitivity to ET. Among several QOL modules, we chose FACT-ES as a suitable PRO during ET. When interpreting HR-QOL data, it is important to consider clinically meaningful absolute score differences. However, to date, no study has estimated MIDs in patients with MBC. Additionally, neither the MIDs for FACT-ES nor those of the Japanese cohort for FACT-B have been investigated. Therefore, this is the first study in which the MIDs of the HR-QOL score are estimated. MIDs are generally estimated using the distribution-based and anchorbased methods [8]. When using the anchor-based method, deterioration and improvement often have different values. In this study, as described in another report [15], The magnitude of the decline of all the HR-QOL scores was larger than that of the improvement of the HR-QOL scores. One reason for this discrepancy is 'response bias' [14]. Specifically, many cancer patients tend to feel improvement even in small changes, as they value the duration and cost of treatment.

Regarding HR-QOL evaluation for patients with MBC during second-line ET, some studies have shown an improvement in the HR-QOL for patients treated with a combination of ET and targeted therapy [16-18]. However, there is no HR-QOL study for patients with MBC having a low sensitivity to ET during secondline ET focusing on endocrine symptoms. Moreover, there is no data on Japanese patients. Therefore, to discuss the differences in hormone sensitivity and cross-cultural discrepancy in FACT scores, we compared our data with that of previous literature. First, the baseline HR-QOL scores in this study were 
similar to those of the FALCON study, a randomized controlled trial of first-line ET for MBC [19]. Conversely, the baseline score was better than that of the CONFIRM trial, which is a trial of second-line ET for MBC [20]. The reason for this is that more than half of the patients had no visceral metastasis or previous ET did not result in prolonged adverse events. Second, regarding the cross-cultural differences of MID, distribution- and anchor-based MIDs in our data were also similar to those in previous studies [10, 21]. Based on the abovementioned points, we speculate that MIDs of FACT scores are generally within the same range across different treatment settings, cultures, and ethnicities.

For further analyses of the relationship between clinical response and HR-QOL, we defined MIDs of the FACT-ES total score as 8 and 5 points for declined and improved HR-QOL scores based on the distribution- and anchor-based method, respectively. The proportions of patients who experienced MID change in the FACT-ES total score did not differ with the clinical benefit status. However, the proportion of patients with improved MIDs tended to be higher at 1 month in those with clinical benefit than in those without. Further, the proportion of patients with MID deterioration tended to be lower at 3 months in those who experienced clinical benefit than in those who did not. Although the results showed no statistically significant association between MIDs and CBR, the patients who experienced clinical benefit tended to have fewer declines and much more improvement. Moreover, the mean change in the FACT-ES total score from baseline at 1 and 3 months also tended to improve in patients who experienced a clinical benefit. In general, even if clinical benefit is achieved with cytotoxic chemotherapy, the adverse events may deteriorate the HR-QOL. In contrast, since there are fewer side effects of ET, it is conceivable that clinical efficacy correlates with HR-QOL (e.g., non-hematological adverse events of more than grade 3 in this study were very rare, such as fatigue in two patients, depression in one patient, or appetite loss in one patient). Given the 7-month PFS in this study, we hypothesized that an HR-QOL decline within 3 months would be a surrogate for disease progression.

This study had some limitations. First, the sample size was small, and we could not estimate the MIDs in detail based on the seven SSQ categories. Second, the planned duration of HR-QOL surveys is relatively short (only 3 months), to avoid missing data due to long-term survey. Third, the MIDs of FACT-ES were estimated for a limited cohort, such as patients on second-line ET, or those mostly treated with fulvestrant. Additionally, the current standard of care is the combination of ET with cyclin-dependent kinase 4/6 inhibitors. Finally, the relationship between OS and HR-QOL is unclear because the primary outcome of the main paper was CBR. However, this study stands out in that the MIDs of FACT-ES were estimated using the SSQ prospectively, which has not been published before. Further, we have reaffirmed the importance of assessing HR-QOL during the treatment of patients with MBC.

In conclusion, there were no apparent cultural differences in the FACT scores between Japanese patients and patients from other countries. For the first time in literature, the MIDs of the FACT-ES total score were estimated to be 5 for improvement and 8 for decline. Patients who were able to maintain their HR-QOL during the first 3 months from the start of second-line ET were likely to experience clinical benefits. Therefore, evaluating FACT-ES may serve as a surrogate for the effectiveness of second-line ET for patients with MBC having a low sensitivity to ET. Based on our results, early imaging examination and 
transition to the next sequential therapy such as chemotherapy may be indicated in patients with decreased FACT-ES scores. Further studies with larger sample sizes and current standards of care, such as ET with some targeted therapies are warranted.

\section{Declarations}

\section{Acknowledgements}

This study was conducted as a research support project of the General Incorporated Association of Comprehensive Support Project for Oncological Research of Breast Cancer (CSPOR-BC).

We thank Dr. T. Yamaguchi and Dr. T. Shimoi for their valuable comments on this paper.

\section{Funding}

This study was funded by AstraZeneca.

\section{Compliance with ethical standards}

\section{Conflict of Interest}

Yuichiro Kikawa received honoraria from Eisai, Novartis, Pfizer, Lilly, Taiho, and Chugai, outside the submitted work. Masato Takahashi received grants from Kyowa Hakko Kirin and Taiho, personal fees from AstraZeneca, Eisai, Eli Lilly, and Pfizer, outside the submitted work. Tatsuya Toyama received grants and personal fees from Chugai, Eisai, Novartis, Takeda, Nippon Kayaku, Pfizer, Lilly, and Daiichi Sankyo, and personal fees from AstraZeneca, and grants from Taiho and Kyowa Kirin, outside the submitted work. Hirofumi Mukai received honoraria from Pfizer, Takeda, Daiichi Sankyo, and Taiho, and grants from the Japanese government, Daiichi Sankyo, and Pfizer, outside the submitted work. The remaining authors have no conflicts of interest to disclose.

\section{Ethical approval}

All procedures were performed in accordance with the Helsinki declaration and the ethical standards of the institutional research committee. Informed consent was obtained from all study participants.

\section{Author contributions}

Yuichiro Kikawa: Data acquisition, writing-original draft, and writing revisions. Yasuhiro Hagiwara: Data curation and analysis, writing-original draft, and writing review. Tomomi Fujisawa: Conceptualization, data acquisition, and writing review. Kazuhiro Araki, Takayuki Iwamoto, Takafumi Sangai, Tadahiko Shien, Shintaro Takao, and Nishimura: Data acquisition and writing review. Masato Takahashi, Tatsuya Toyama, Tomohiko Aihara, and Hirofumi Mukai: Project administration and writing review. Naruto Taira: Conceptualization, project administration, writing-original draft, and writing review. 


\section{References}

1. Hortobagyi GN (1998) Treatment of breast cancer. N Engl J Med 339:974-984. doi:10.1056/nejm199810013391407

2. Cardoso F, Senkus E, Costa A et al (2018) 4th ESO-ESMO International Consensus Guidelines for Advanced Breast Cancer (ABC 4)†. Ann Oncol 29:1634-1657. doi:10.1093/annonc/mdy192

3. Rugo HS, Rumble RB, Macrae E et al (2016) Endocrine Therapy for Hormone Receptor-Positive Metastatic Breast Cancer: American Society of Clinical Oncology Guideline. J Clin Oncol 34:30693103. doi:10.1200/jco.2016.67.1487

4. Iwamoto T, Fujisawa T, Shien T et al (2020) The efficacy of sequential second-line endocrine therapies (ETs) in postmenopausal estrogen receptor-positive and HER2-negative metastatic breast cancer patients with lower sensitivity to initial ETs. Breast Cancer 27:973-981. doi:10.1007/s12282020-01095-y

5. Cella DF (1995) Measuring quality of life in palliative care. Semin Oncol 22:73-81

6. Guyatt GH, Feeny DH, Patrick DL (1993) Measuring health-related quality of life. Ann Intern Med 118:622-629. doi:10.7326/0003-4819-118-8-199304150-00009

7. King MT (2011) A point of minimal important difference (MID): a critique of terminology and methods. Expert Rev Pharmacoecon Outcomes Res 11:171-184. doi:10.1586/erp.11.9

8. Revicki D, Hays RD, Cella D, Sloan J (2008) Recommended methods for determining responsiveness and minimally important differences for patient-reported outcomes. J Clin Epidemiol 61:102-109. doi:10.1016/j.jclinepi.2007.03.012

9. Osoba D, Rodrigues G, Myles J, Zee B, Pater J (1998) Interpreting the significance of changes in health-related quality-of-life scores. J Clin Oncol 16:139-144. doi:10.1200/jco.1998.16.1.139

10. Eton DT, Cella D, Yost KJ et al (2004) A combination of distribution- and anchor-based approaches determined minimally important differences (MIDs) for four endpoints in a breast cancer scale. J Clin Epidemiol 57:898-910. doi:10.1016/j.jclinepi.2004.01.012

11. Musoro JZ, Coens C, Fiteni F et al (2019) Minimally Important Differences for Interpreting EORTC QLQ-C30 Scores in Patients With Advanced Breast Cancer. JNCI Cancer Spectr 3(3):pkz037. doi:10.1093/jncics/pkz037

12. Fallowfield LJ, Leaity SK, Howell A, Benson S, Cella D (1999) Assessment of quality of life in women undergoing hormonal therapy for breast cancer: validation of an endocrine symptom subscale for the FACT-B. Breast Cancer Res Treat 55:189-199. doi:10.1023/a:1006263818115

13. Brady MJ, Cella DF, Mo F et al (1997) Reliability and validity of the Functional Assessment of Cancer Therapy-Breast quality-of-life instrument. J Clin Oncol 15:974-986. doi:10.1200/jco.1997.15.3.974

14. Cella DF, Tulsky DS, Gray G et al (1993) The Functional Assessment of Cancer Therapy scale: development and validation of the general measure. J Clin Oncol 11:570-579. doi:10.1200/JC0.1993.11.3.570 
15. Cella D, Hahn EA, Dineen K (2002) Meaningful change in cancer-specific quality of life scores: differences between improvement and worsening. Qual Life Res 11:207-221. doi:10.1023/a:1015276414526

16. Burris HA 3rd, Lebrun F, Rugo HS et al (2013) Health-related quality of life of patients with advanced breast cancer treated with everolimus plus exemestane versus placebo plus exemestane in the phase 3, randomized, controlled, BOLERO-2 trial. Cancer 119:1908-1915. doi:10.1002/cncr.28010

17. Fribbens C, O'Leary B, Kilburn L et al (2016) Plasma ESR1 Mutations and the Treatment of Estrogen Receptor-Positive Advanced Breast Cancer. J Clin Oncol 34:2961-2968. doi:10.1200/jco.2016.67.3061

18. Harbeck N, lyer S, Turner N et al (2016) Quality of life with palbociclib plus fulvestrant in previously treated hormone receptor-positive, HER2-negative metastatic breast cancer: patient-reported outcomes from the PALOMA-3 trial. Ann Oncol 271047-271054. doi:10.1093/annonc/mdw139

19. Robertson JFR, Cheung K-L, Noguchi S et al (2018) Health-related quality of life from the FALCON phase III randomised trial of fulvestrant $500 \mathrm{mg}$ versus anastrozole for hormone receptor-positive advanced breast cancer. Eur J Cancer 94:206-215. doi:10.1016/j.ejca.2018.02.026

20. Di Leo A, Jerusalem G, Petruzelka L et al (2010) Results of the CONFIRM phase III trial comparing fulvestrant $250 \mathrm{mg}$ with fulvestrant $500 \mathrm{mg}$ in postmenopausal women with estrogen receptorpositive advanced breast cancer. J Clin Oncol 28:4594-4600. doi:10.1200/jco.2010.28.8415

21. Ringash J, O'Sullivan B, Bezjak A, Redelmeier DA (2007) Interpreting clinically significant changes in patient-reported outcomes. Cancer 110:196-202. doi:10.1002/cncr.22799

\section{Tables}

Table 1. Patient characteristics at baseline $(n=47)$ 


\begin{tabular}{ll}
\hline Variable & Value \\
\hline Age (median and IQR) & $66(61-72)$ \\
ECOG performance status, n (\%) & $39(83.0)$ \\
0 & $7(14.9)$ \\
1 & $1(2.1)$ \\
2 & $23(48.9)$ \\
Presence of visceral metastasis, n (\%) & $39(83.0)$ \\
Second-line endocrine therapy, n (\%) & $4(8.5)$ \\
Fulvestrant & $1(2.1)$ \\
Selective estrogen receptor modulator & $3(6.4)$ \\
Aromatase inhibitor & \\
mTOR inhibitor plus aromatase inhibitor & $23(48.9)$ \\
Marital status, n (\%) & $5(10.6)$ \\
Married or with partner & $11(23.4)$ \\
Never married & $8(17.0)$ \\
Separated or divorced & \\
Widowed & $5(10.6)$ \\
Education level, n (\%) & $31(66.0)$ \\
9 years or less & $10(21.3)$ \\
10 to 12 years & $1(2.1)$ \\
13 years or more & \\
Missing & $4(8.5)$ \\
Employment status at baseline, n (\%) & $10(21.3)$ \\
Full time & $16(34.0)$ \\
Part time & $3(6.4)$ \\
Homemaker & $14(29.8)$ \\
Retired & \\
Unemployed & $12(25.5)$ \\
Living situation (multiple answers allowed), n (\%) & $24(51.1)$ \\
Alone & $18(38.3)$ \\
With husband & $6(12.8)$ \\
With children & $9(19.1)$ \\
With parents & \\
Others & \\
\hline
\end{tabular}

IQR, interquartile range; ECOG, Eastern Cooperative Oncology Group; mTOR, mammalian target of rapamycin.

Table 2. Summary of HR-QOL scores and MID estimates based on the distribution method 


\begin{tabular}{|c|c|c|c|c|c|c|c|}
\hline & \multirow[b]{2}{*}{$\begin{array}{l}\text { Number of responded/ } \\
\text { expected }\end{array}$} & \multirow[b]{2}{*}{ Number of } & \multirow[b]{2}{*}{ Mean (SD) } & \multirow[b]{2}{*}{$\begin{array}{l}\text { Cronbach's } \\
\alpha\end{array}$} & \multicolumn{3}{|l|}{$\begin{array}{l}\text { MID } \\
\text { estimate }\end{array}$} \\
\hline & & & & & $1 / 2 \mathrm{SD}$ & $\begin{array}{l}1 / 3 \\
\mathrm{SD}\end{array}$ & SEM \\
\hline FACT-G total & & & & & & & \\
\hline Baseline & $47 / 47$ (100\%) & & $75.1(14.7)$ & 0.85 & 7.3 & 4.9 & 5.7 \\
\hline 1 month & $43 / 47(91.5 \%)$ & & 74.9 (13.8) & 0.85 & 6.9 & 4.6 & 5.4 \\
\hline 3 months & $43 / 47$ (91.5\%) & & $74.6(16.1)$ & 0.90 & 8.1 & 5.4 & 5.1 \\
\hline Weighted & - & & - & - & 7.4 & 5.0 & 5.4 \\
\hline \multicolumn{8}{|l|}{$\begin{array}{l}\text { mean } \\
\text { FACT-G TOI }\end{array}$} \\
\hline Baseline & $47 / 47(100 \%)$ & & $41.5(9.0)$ & 0.85 & 4.5 & 3.0 & 3.5 \\
\hline 1 month & $44 / 47(93.6 \%)$ & & $41.9(8.5)$ & 0.86 & 4.2 & 2.8 & 3.2 \\
\hline 3 months & 43/47 (91.5\%) & & $40.8(9.9)$ & 0.89 & 5.0 & 3.3 & 3.2 \\
\hline Weighted & - & & - & - & 4.6 & 3.0 & 3.3 \\
\hline \multicolumn{8}{|l|}{$\begin{array}{l}\text { mean } \\
\text { FACT-B total }\end{array}$} \\
\hline Baseline & $47 / 47$ (100\%) & & 100.2 & 0.86 & 8.8 & 5.9 & 6.7 \\
\hline 1 month & 43/47 (91.5\%) & & 100.4 & 0.84 & 7.9 & 5.3 & 6.3 \\
\hline 3 months & 43/47 (91.5\%) & & 99.8 (19.3) & 0.89 & 9.6 & 6.4 & 6.3 \\
\hline Weighted & - & & - & - & 8.8 & 5.9 & 6.4 \\
\hline \multirow{2}{*}{\multicolumn{8}{|c|}{$\begin{array}{l}\text { mean } \\
\text { FACT-B TOI }\end{array}$}} \\
\hline & & & & & & & \\
\hline Baseline & 47/47 (100\%) & & 66.6 (12.5) & 0.84 & 6.2 & 4.2 & 5.0 \\
\hline 1 month & 44/47 (93.6\%) & & 67.1 (11.8) & 0.84 & 5.9 & 3.9 & 4.8 \\
\hline 3 months & 43/47 (91.5\%) & & 66.1 (13.6) & 0.87 & 6.8 & 4.5 & 4.9 \\
\hline mean Weighted & - & & - & - & 6.3 & 4.2 & 4.9 \\
\hline \multicolumn{8}{|l|}{ FACT-ES total } \\
\hline Baseline & $47 / 47$ (100\%) & & 140.9 & 0.85 & 9.1 & 6.1 & 7.1 \\
\hline 1 month & 43/47 (91.5\%) & & 140.7 & 0.85 & 8.5 & 5.7 & 6.6 \\
\hline 3 months & $43 / 47$ (91.5\%) & & $\begin{array}{l}140.1 \\
(20.4)\end{array}$ & 0.90 & 10.2 & 6.8 & 6.4 \\
\hline mean ${ }^{\text {Weighted }}$ & - & & - & - & 9.2 & 6.2 & 6.7 \\
\hline \multicolumn{8}{|l|}{ FACT-ES TOI } \\
\hline Baseline & $47 / 47$ (100\%) & & $\begin{array}{l}107.3 \\
(13.3)\end{array}$ & 0.83 & 6.7 & 4.4 & 5.4 \\
\hline 1 month & 44/47 (93.6\%) & & 107.3 & 0.85 & 6.8 & 4.5 & 5.2 \\
\hline 3 months & 43/47 (91.5\%) & & $\begin{array}{l}106.3 \\
(15.0)\end{array}$ & 0.88 & 7.5 & 5.0 & 5.2 \\
\hline Weighted & - & & - & - & 7.0 & 4.6 & 5.3 \\
\hline \multicolumn{8}{|l|}{$\begin{array}{l}\text { mean } \\
\text { ESS }\end{array}$} \\
\hline Baseline & $47 / 47$ (100\%) & & 65.8 (7.9) & 0.77 & 4.0 & 2.6 & 3.8 \\
\hline 1 month & 43/47 (91.5\%) & & $65.3(8.0)$ & 0.78 & 4.0 & 2.7 & 3.8 \\
\hline 3 months & 43/47 (91.5\%) & & 65.7 (7.9) & 0.79 & 4.0 & 2.6 & 3.7 \\
\hline mean Weighted & - & & - & - & 4.0 & 2.7 & 3.7 \\
\hline
\end{tabular}

HR-QOL, health-related quality of life; MID, minimally important difference; SD, standard deviation; SEM, standard error of measurement; FACT, Functional Assessment of Cancer Therapy; G, general; TOI, trial outcome index; B, breast; ES, endocrine symptom; ESS, endocrine symptom subscale.

The weighted mean was calculated using the number of scores at each time point as weight.

Table 3. Liner spline MID estimates based on the anchor method 


\begin{tabular}{|c|c|c|}
\hline & Estimate & Difference (vs stable) \\
\hline \multicolumn{3}{|l|}{ FACT-G total } \\
\hline Moderately worse & -15.2 & -14.9 \\
\hline A little worse & -7.7 & -7.5 \\
\hline About the same & -0.3 & - \\
\hline A little better & 2.9 & 3.1 \\
\hline Moderately better & 6.0 & 6.3 \\
\hline \multicolumn{3}{|l|}{ FACT-G TOI } \\
\hline Moderately worse & -13.6 & -14.0 \\
\hline A little worse & -6.6 & -7.0 \\
\hline About the same & 0.4 & - \\
\hline A little better & 3.0 & 2.6 \\
\hline Moderately better & 5.6 & 5.2 \\
\hline \multicolumn{3}{|l|}{ FACT-B total } \\
\hline Moderately worse & -15.9 & -15.9 \\
\hline A little worse & -8.0 & -7.9 \\
\hline About the same & -0.1 & - \\
\hline A little better & 2.4 & 2.5 \\
\hline Moderately better & 4.9 & 4.9 \\
\hline \multicolumn{3}{|l|}{ FACT-B TOI } \\
\hline Moderately worse & -14.4 & -14.9 \\
\hline A little worse & -7.0 & -7.4 \\
\hline About the same & 0.5 & - \\
\hline A little better & 2.5 & 2.0 \\
\hline \multirow{2}{*}{\multicolumn{3}{|c|}{ FACT-ES total }} \\
\hline & & \\
\hline Moderately worse & -16.6 & -16.7 \\
\hline A little worse & -8.3 & -8.3 \\
\hline About the same & 0.0 & - \\
\hline A little better & 2.0 & 2.0 \\
\hline Moderately better & 4.0 & 3.9 \\
\hline \multicolumn{3}{|l|}{ FACT-ES TOI } \\
\hline Moderately worse & -15.1 & -15.7 \\
\hline A little worse & -7.3 & -7.8 \\
\hline About the same & 0.6 & - \\
\hline A little better & 2.0 & 1.5 \\
\hline Moderately better & 3.5 & 3.0 \\
\hline \multicolumn{3}{|l|}{ ESS } \\
\hline Moderately worse & -8.2 & -8.2 \\
\hline A little worse & -4.1 & -4.1 \\
\hline About the same & 0.0 & - \\
\hline A little better & -0.3 & -0.3 \\
\hline Moderately better & -0.6 & -0.6 \\
\hline
\end{tabular}

MID: minimally important difference; FACT: functional assessment of cancer therapy; G: general; TOI: trial outcome index; B: breast; ES: endocrine symptom; ESS: endocrine symptom subscale.

Table 4. Three-category MID estimates based on the anchor method 


\begin{tabular}{|c|c|c|c|c|}
\hline \multirow[t]{2}{*}{ Three-category changes from baseline } & \multicolumn{2}{|c|}{ The number of patients } & \multirow[t]{2}{*}{ Estimate } & \multirow[t]{2}{*}{ Difference (vs stable) } \\
\hline & at 1 month & at 3 months & & \\
\hline \multicolumn{5}{|l|}{ FACT-G total } \\
\hline Decline & 4 & 10 & -8.2 & -7.8 \\
\hline Stable & 27 & 22 & -0.4 & \\
\hline Improve & 10 & 9 & 4.4 & 4.7 \\
\hline \multicolumn{5}{|l|}{ FACT-G TOI } \\
\hline Decline & 4 & 10 & -7.1 & -7.3 \\
\hline Stable & 28 & 22 & 0.3 & \\
\hline Improve & 10 & 9 & 4.2 & 4.0 \\
\hline \multicolumn{5}{|l|}{ FACT-B total } \\
\hline Decline & 4 & 10 & -7.9 & -7.3 \\
\hline Stable & 27 & 22 & -0.5 & \\
\hline Improve & 10 & 9 & 3.9 & 4.4 \\
\hline \multicolumn{5}{|l|}{ FACT-B TOI } \\
\hline Decline & 4 & 10 & -6.8 & -6.8 \\
\hline Stable & 28 & 22 & 0.0 & \\
\hline Improve & 10 & 9 & 3.8 & 3.8 \\
\hline \multicolumn{5}{|l|}{ FACT-ES total } \\
\hline Decline & 4 & 10 & -8.2 & -7.7 \\
\hline Stable & 27 & 22 & -0.5 & - \\
\hline Improve & 10 & 9 & 3.6 & 4.1 \\
\hline \multicolumn{5}{|l|}{ FACT-ES TOI } \\
\hline Decline & 4 & 10 & -7.2 & -7.2 \\
\hline Stable & 28 & 22 & 0.0 & \\
\hline Improve & 10 & 9 & 3.4 & 3.4 \\
\hline \multicolumn{5}{|l|}{ ESS } \\
\hline Decline & 2 & 3 & -4.1 & -4.1 \\
\hline Stable & 38 & 32 & 0.0 & - \\
\hline Improve & 2 & 5 & -0.5 & -0.5 \\
\hline
\end{tabular}

MID: minimally important difference; FACT: functional assessment of cancer therapy; G: general; TOI: trial outcome index; B: breast; ES: endocrine symptom; ESS: endocrine symptom subscale. $\mathrm{n}$ represents sum of the number of scores at 1 and 3 months.

\section{Figures}


Fig. 1

a

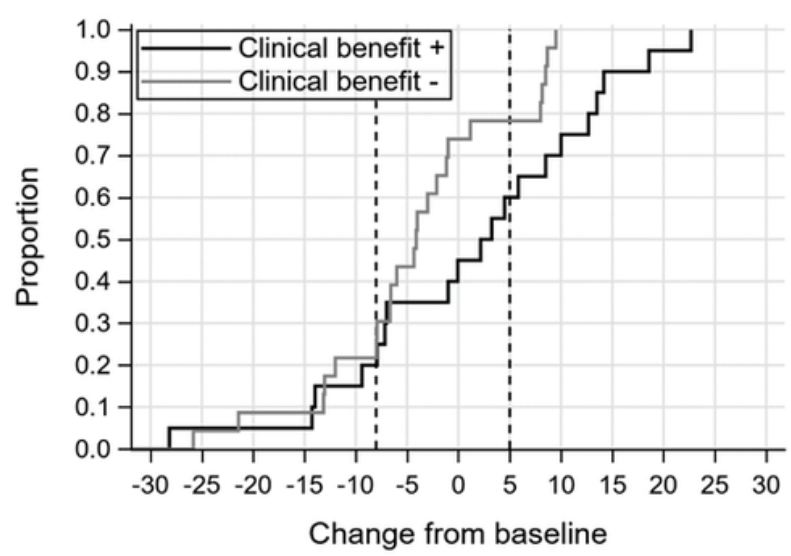

b

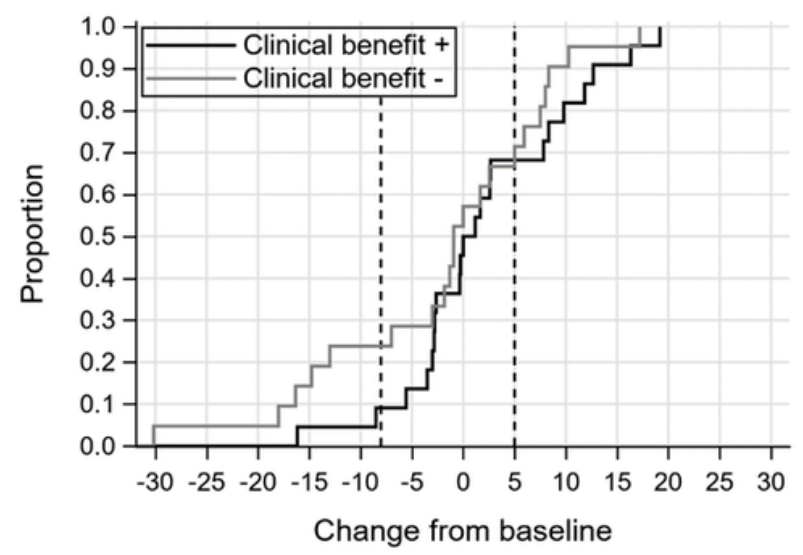

\section{Figure 1}

(a) The cumulative distribution functions for the FACT-ES total score according to the clinical benefit status at 1 month. The vertical dotted lines indicate the MID thresholds. (b) The cumulative distribution functions for the FACT-ES total score according to the clinical benefit status at 3 months. The vertical dotted lines indicate the MID thresholds. 
Fig. 2

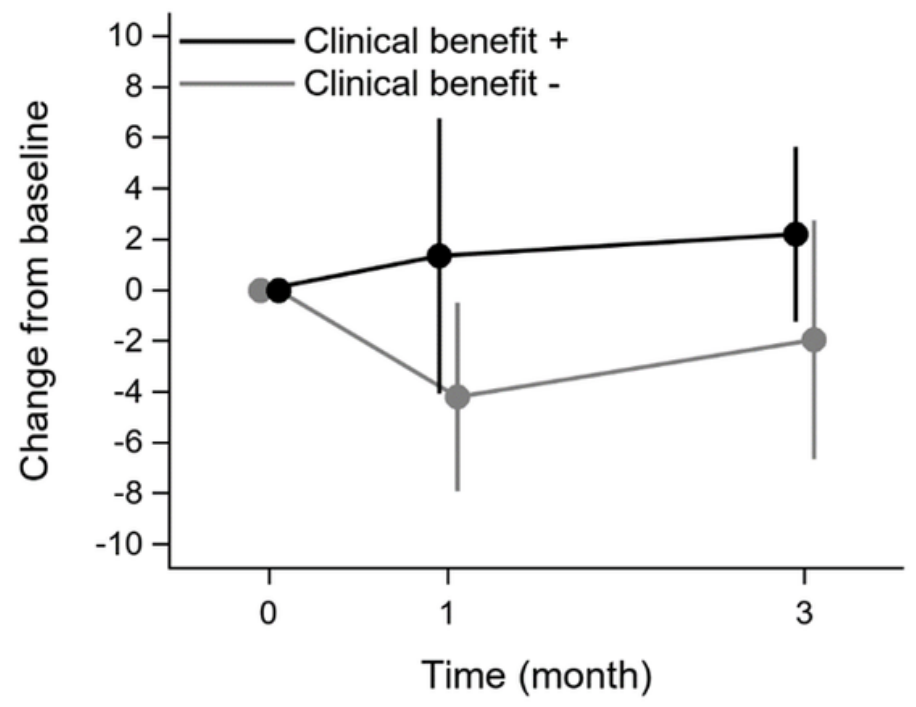

Figure 2

The mean changes in the FACT-ES total score from baseline according to clinical benefit status. 